PROCEEDINGS OF THE

AMERICAN MATHEMATICAL SOCIETY

Volume 124, Number 12, December 1996, Pages 3637-3640

S 0002-9939(96)03584-8

\title{
ON RATIONAL INVARIANTS OF THE GROUP $E_{6}$
}

\author{
A. V. ILTYAKOV
}

(Communicated by Eric Friedlander)

\begin{abstract}
We prove rationality of the field of invariants in several variables of a minimal irreducible representation of a simple algebraic group of type $E_{6}$ over an algebraically closed field of characteristic zero.
\end{abstract}

This note completes the main result of [3], where the reader can find an extensive introduction and basic notations.

Let $\mathbf{A}=H\left(\mathbf{O}_{3}\right)$ be an Albert algebra [4, p.21] over an algebraically closed field $F$ of characteristic 0 . Any element $x \in \mathbf{A}$ satisfies a cubic equation over $F$

$$
x^{3}-x^{2} t_{1}(x)+x t_{2}(x)-e t_{3}(x)=0,
$$

where $e$ is the unit of $\mathbf{A}, t_{1}(x)$ is the trace $\operatorname{tr}(x)$ of $x$ and $t_{3}(x)$ is the cubic norm $N(x)$ of $x$.

The group of norm-preserving linear transformations

$$
M=\{g \in \operatorname{End}(\mathbf{A}) \mid g \text { is invertible, } \forall x \in \mathbf{A} N(x \cdot g)=N(x)\}
$$

is a simple algebraic group of type $E_{6}[1]$ and $\mathbf{A}$ is an irreducible $M$-module of dimension 27 [5, th.14]. The form $\operatorname{tr}(x y)$ is nondegenerate. For $g \in \operatorname{End}(\mathbf{A})$ denote by $g^{*}$ the transposed transformation with respect to this form. We may consider $\mathbf{A}$ as an $M$-module $\mathbf{A}^{*}$ under the action $x \rightarrow x \cdot g^{\tau}$, where $g \rightarrow g^{\tau}=\left(g^{*}\right)^{-1}$ is an (outer) automorphism of $M\left[4\right.$, p.370, ex.2] (in other words, $\mathbf{A}^{*}$ is the module contragredient to $\mathbf{A})$. Let $\mathbf{V}_{k, m}=k \mathbf{A} \oplus m \mathbf{A}^{*}$, where $k \mathbf{A}\left(m \mathbf{A}^{*}\right)$ is the direct sum of $k(m)$ copies of $\mathbf{A}\left(\mathbf{A}^{*}\right)$ and $M$ acts diagonally.

Theorem 1. For any $k, m \geq 0$ the field of rational invariants $F\left(\mathbf{V}_{k, m}\right)^{M}$ is purely transcendental over $F$.

Proof. Since $F\left(\mathbf{V}_{k, m}\right)^{M} \simeq F\left(\mathbf{V}_{m, k}\right)^{M}$, we can assume that $k \geq 1$. Using the slice method [2] (see also, for example, [8]), we shall show that this field is isomorphic to a simple transcendental extension of $F(n \mathbf{A})^{G}$, where $n=m+k-1$ and $G$ is the group of automorphisms $A u t(\mathbf{A})$. Since rationality of the latter field is obtained in [3], this will prove the statement. In particular, the transcendence degree of this

Received by the editors June 8, 1995.

1991 Mathematics Subject Classification. Primary 20G15; Secondary 17C20.

Key words and phrases. Algebraic groups, invariant theory.

During 1993/94 a Becario de FICYT at the University of Oviedo, Asturias, Spain.

(C)1996 American Mathematical Society 
field is computed in the following way (cf. [3]):

$$
\operatorname{tr.} \operatorname{deg}_{F} F\left(\mathbf{V}_{k, m}\right)^{M}= \begin{cases}30+27(m+k-4), & m+k \geq 4, \\ 11, & m+k=3, \\ 3, & m+k=2, \\ 1, & m+k=1\end{cases}
$$

Lemma 1 ([5, th.7], [4, p.244]). 1 . If $a \in \mathbf{A}$ and $N(a)=1$, then there is $\eta \in M$ such that $a \cdot \eta=e$.

2. If $\phi \in M$ and $e \cdot \phi=e$, then $\phi$ is an automorphism of $\mathbf{A}$.

From the lemma it follows that the subspace

$$
E=\left\{\bar{a}=\left(a_{1}, \ldots, a_{k}, b_{1}, \ldots, b_{m}\right) \in \mathbf{V}_{k, m} \mid a_{k} \in e F\right\}
$$

is a slice of the $M$-module $\mathbf{V}_{k, m}$ [2, sect.3]. Hence, $F\left(\mathbf{V}_{k, m}\right)^{M} \simeq F(E)^{N(E)}$, where $N(E)=\{\phi \in M \mid E \cdot \phi \subseteq E\}$. Also, $N(E)=G \times C_{3}$, where $C_{3}$ is the group of linear transformations $R_{x}: y \rightarrow y x$, where $x=e \xi, \xi \in F$ and $\xi^{3}=1$.

Notice that $E=e F \oplus \mathbf{V}_{k-1, m}$. Denote by $t$ the coordinate function of the first summand and by $y_{i}\left(z_{i}\right)$ the projector from $k \mathbf{A}\left(m \mathbf{A}^{*}\right)$ onto the $i$-th summand (generic elements of $\mathbf{A}[3])$. Consider a monomorphism $\psi: F\left(\mathbf{V}_{k-1, m}\right) \rightarrow F(E)$ : if $f=f\left(y_{2}, \ldots, y_{k}, z_{1}, \ldots, z_{m}\right)$, then $\psi(f)=f\left(y_{2} / t, \ldots, y_{k} / t, z_{1} t, \ldots, z_{m} t\right)$. Since $R_{x}^{\tau}=R_{x^{-1}}$ for $R_{x} \in C_{3}$, the image $K$ of $F\left(\mathbf{V}_{k-1, m}\right)^{G}$ lies in $F(E)^{N(E)}=$ $\left(F(E)^{G}\right)^{C_{3}}$. Observe that $F(E)$ can be considered as the field of rational functions of the $G$-module $\mathbf{V}_{k-1, m} \otimes_{F} F(t)$ over $F(t)$, where the generic elements are $y_{2} / t, \ldots, y_{k} / t, z_{1} t, \ldots, z_{m} t$; hence, $F(E)^{G}=K(t)$. Since for the extension degree we have $\left|F(E)^{N(E)}: F(E)^{G}\right|=3$, we get $K\left(t^{3}\right)=F(E)^{N(E)} \simeq F\left(\mathbf{V}_{k, m}\right)^{M}$.

It is known that the field of invariants $F(k \mathbf{A})^{G}$ is generated by trace polynomials [3]. Working out this proof, we shall describe generators of the field $F\left(\mathbf{V}_{k, m}\right)^{M}$ in a similar way. Let $N(x, y, z)$ be the linearization of $N(x)(N(x, x, x)=3 N(x))$ and let $x \rightarrow x^{\#}$ be a quadratic operation such that $\operatorname{tr}\left(x^{\#} y\right)=N(x, x, y)[4,6]$. Also, $x \times y=(x+y)^{\#}-x^{\#}-y^{\#}$. Consider a pair of subspaces $\Delta, \Delta^{*} \subseteq \mathbf{A} \otimes_{F} F\left[\mathbf{V}_{k, m}\right]$ which are minimal with the following properties:

1. $y_{1}, \ldots, y_{k} \in \Delta, z_{1}, \ldots, z_{m} \in \Delta^{*}$;

2. if $u, v \in \Delta\left(u, v \in \Delta^{*}\right)$, then $u \times v \in \Delta^{*}(\Delta)$;

Theorem 2. The field $F\left(\mathbf{V}_{k, m}\right)^{M}$ is generated by elements $N(u), u \in \Delta$.

By the definition of $x \rightarrow x^{\#}$, for any $g \in M$ we have $(x \cdot g)^{\#}=x^{\#} \cdot g^{\tau}$. The next statement can be easily checked by induction.

Lemma 2. Let $u \in \Delta\left(\Delta^{*}\right)$. Then for any $g \in M$ the polynomial

$$
u\left(y_{1} \cdot g, \ldots, y_{k} \cdot g, z_{1} \cdot g^{\tau}, \ldots, z_{m} \cdot g^{\tau}\right)
$$

is equal to $u \cdot g$ (respectively, $\left.u \cdot g^{\tau}\right)$.

In particular, this yields $N(u) \in F\left(\mathbf{V}_{k, m}\right)^{M}$ for any $u \in \Delta$. Since $M$ is a simple linear algebraic group, the field $F\left(\mathbf{V}_{k, m}\right)^{M}$ is generated by homogeneous polynomial invariants [7, sect.3.3 ]. Take such an invariant $f=f\left(y_{1}, \ldots, y_{k}, z_{1}, \ldots, z_{m}\right)$ and put $p=\sum_{i=1}^{k} \operatorname{deg}_{y_{i}}(f), q=\sum_{i=1}^{m} \operatorname{deg}_{z_{i}}(f)$. Recall that $E$ is a slice and, therefore, the mapping $\pi:\left.f \rightarrow f\right|_{E}$ is a monomorphism from the algebra of polynomial invariants $F\left[\mathbf{V}_{k, m}\right]^{M}$ to $F(E)^{N(E)}$ and its image generates this field. Denote $\Omega=$ 
$\operatorname{alg}_{F}\{N(u) \mid u \in \Delta\} \subseteq F\left[\mathbf{V}_{k, m}\right]^{M}$. To prove the theorem it suffices to show that $\pi(f)$ belongs to the field $K$ generated by $\pi(\Omega)$.

Observe that $t^{3}=\pi\left(N\left(y_{1}\right)\right) \in K$. Also, $\pi(f)=h t^{p-q}$, where

$$
h=f\left(e, y_{2} / t, \ldots, y_{k} / t, z_{1} t, \ldots, z_{m} t\right)=\psi(\tilde{f})
$$

and $\tilde{f}=\left.f\right|_{y_{1}=e} \in F\left(\mathbf{V}_{k-1, m}\right)^{G}$. Recall that $\psi(\tilde{f}) \in F(E)^{N(E)}$; hence, $t^{p-q}$ also lies in this field. Therefore, $r=(p-q) / 3$ is an integer and $t^{p-q} \in K$. In particular, $\psi(\tilde{g})$ is defined and belongs to $K$ for any $g \in \Omega$ and, therefore, for any $g \in F(\Omega)$. If we show that $\tilde{f}=\tilde{g}$ for some $g \in F(\Omega)$, then we shall get $h=\psi(\tilde{g}) \in K$ and prove the theorem.

The field $F\left(\mathbf{V}_{k-1, m}\right)^{G}$ is generated by polynomials $\operatorname{tr}(u)$, where $u \in$ $B_{k+m-1}=\operatorname{alg}_{F}\left\{y_{2}, \ldots, y_{k}, z_{1}, \ldots, z_{m}\right\}$ [3]. It suffices to show that $\operatorname{tr}(u)=\tilde{g}$ for an appropriate $g \in F(\Omega)$. Let $\Delta \Omega$ be the $\Omega$-module generated by $\Delta$.

Lemma 3. For any $u \in B_{k+m-1}$ there is $h_{u} \in \Delta \Omega$ such that $\left.h_{u}\right|_{y_{1}=e}=u$.

Proof. We shall need the following identities, which follow from [6, eq.(11),(21)]:

$$
\begin{gathered}
a=-a \times e+\operatorname{tr}(a) \cdot e, \\
a^{2}=(a \times e)^{\#}-N(a, a, e) e .
\end{gathered}
$$

Denote $\omega(a, b)=-a \times b^{\#}+N\left(a, b^{\#}, b^{\#}\right) b$. By (2), if $a \in \Delta^{*}$ and $b \in \Delta$, then $\omega(a, b) \in \Delta \Omega$ and $\omega(a, e)=a$. We assume that $u$ is a monomial and carry out an induction on its degree. First, put $h_{y_{i}}=y_{i}, 2 \leq i \leq k$, and $h_{z_{l}}=\omega\left(z_{l}, y_{1}\right)$, $1 \leq l \leq m$. Now, suppose that $\operatorname{deg}(u)=2$, i.e. $u$ has the form $u_{1}=y_{i} y_{j}, u_{2}=z_{s} y_{j}$ or $u_{3}=z_{s} z_{t}$. Using the linearized form of (3), we may put

$$
h_{u_{1}}=1 / 2\left(y_{i} \times y_{1}\right) \times\left(y_{j} \times y_{1}\right)-N\left(y_{i}, y_{j}, y_{1}\right) y_{1} .
$$

Next,

$$
h_{u_{2}}=\left.h_{u_{1}}\right|_{y_{i}=\omega\left(z_{s}, y_{1}\right)}, \quad h_{u_{3}}=\left.h_{u_{2}}\right|_{y_{j}=\omega\left(z_{t}, y_{1}\right)} .
$$

We can assume that the lemma holds for an arbitrary monomial $u \in B_{k+m-1}$ of degree $<n$ and for arbitrary $k, m$. Every monomial $u, \operatorname{deg}(u)=n \geq 3$, can be represented in the form $u=\left.v\right|_{y_{k+1}=w}$, where $\operatorname{deg}(v), \operatorname{deg}(w)<n$. Hence,

$$
h_{u}=\left.h_{v}\right|_{y_{k+1}=h_{w}} .
$$

To conclude the proof of Theorem 2 we may put $g=N\left(h_{u}, y_{1}, y_{1}\right)$.

Recall that $\Omega$ lies in the algebra of polynomial invariants $F\left[\mathbf{V}_{k, m}\right]^{M}$, and it looks probable that this algebra is generated by elements $N(u), N(v), \operatorname{tr}(u v)$, where $u \in \Delta, v \in \Delta^{*}$.

\section{ACKNOWLEDGMENTS}

The author thanks A.Elduque and the referee for a useful remark which greatly reduced the original proof of Theorem 1 . 


\section{REFERENCES}

1. C. Chevalley, Sur certains groups simples, Tohoku Math. J., 7(1955), pp. 14-66. MR 17:457c

2. Igor V. Dolgachev, Rationality of fields of invariants, Proc. Symp. Pure Math., 46, part 2, pp. 3-16, AMS, Prov., R.I., 1987. MR 89b:14064

3. A.V. Iltyakov and I.P. Shestakov, Invariants of $F_{4}$ and center of the Albert algebra, J. Algebra, 179 (1996), pp. 838-851. CMP 96:07

4. N. Jacobson, Structure and Representations of Jordan Algebras, Amer. Math. Soc. Colloq. Publ. 39, Providence, 1968. MR 40:4330

5. N. Jacobson, Some groups of transformations defined by Jordan algebras. III, Groups of type $E_{6}$, J.Reine Angew. Math., 207 (1961), pp. 61-85. MR 28:3066

6. K.McCrimmon, The Freudenthal-Springer-Tits constructions of exceptional Jordan algebras, Trans.Amer.Math.Soc., 139 (1969), pp. 495-510. MR 42:6064

7. V.L. Popov, E.B. Vinberg, Invariant Theory, Encyclopaedia of Mathematical Sciences. 55 Algebraic Geometry IV, Springer-Verlag, 1994, 123-284. MR 92d:14010 (Russian original).

8. D.J. Saltman, Invariant fields of linear groups and division algebras, Perspect. in Ring Theory (Antwerpen, 1987), pp. 279-297, NATO Adv.Sci.Inst. Ser.C: Math.Phys.Sci. 233, Kluwer Acad.Publ., Dordrecht, 1988. MR 91f:20053

Institute of Mathematics, Siberian Branch of Russian Academy of Sciences, NovosiBIRSK, 630090, RUSSIA

Current address: School of Mathematics and Statistics, University of Sydney, 2006 New South Wales, Australia 